\title{
EDITORIAL
}

\section{Editorial zum Schwerpunktthema: Herausforderungen und Perspektiven der Lehrkräftequalifizierung}

\section{Editorial to the Focus Topic: Challenges of and Perspectives on Teacher Qualification}

Die Professionalisierung von (angehenden) Lehrkräften in unterschiedlichen Phasen ihrer Ausbildung und Berufstätigkeit (einschließlich der Schulleitungen als pädagogischem Führungspersonal) ist in den vergangenen Jahren ein zentrales Thema auch des allgemeinen öffentlichen Interesses geworden, so im Rahmen der Qualitätsoffensive Lehrerbildung (etwa im Hinblick auf einen verbesserten Theorie-Praxis-Bezug), aber auch im Kontext des bundesweiten Lehrkräftebedarfs sowie spezifischer thematischer Herausforderungen (Inklusion, sexualisierte Gewalt, Digitalisierung etc.). Auch eine Lehr-Lern-Kultur mit neuen interprofessionellen Kooperationsanforderungen, etwa im Kontext ganztagsschulischer Konzepte, stellt neue Herausforderungen an Lehrkräfte. Das bedeutet u.a., dass angehende Lehrkräfte vielfach nicht mehr die Praxis vorfinden, die sie aus der eigenen Schulzeit kennen - und damit gegebenenfalls auch vor für sie unerwarteten beruflichen Anforderungen stehen.

In der ersten Phase der Lehrer*innenbildung wurden in vielen Bundesländern verlängerte Praxisphasen eingeführt - nicht zuletzt in der Hoffnung, dass angehende Lehrkräfte sich im Praxissemester noch einmal verstärkt mit ihrer Berufswahl auseinandersetzen. Inwiefern diese Hoffnung berechtigt ist, diskutiert Raphaela Porsch in ihrem Beitrag zur „Berufswahlüberprüfung in Praxisphasen im Lehramtsstudium“ anhand der Befunde einer Längsschnittuntersuchung. Dass in Langzeitpraktika allein schon durch erfahrungsbasierte Einblicke in den beruflichen Alltag des Lehrerberufs individuell-biographisch eine Berufswahlüberprüfung stattfindet, ist erwartbar. Umso bedeutsamer aber sind die im Beitrag herausgearbeiteten Unterschiede mit Blick auf mögliche Quellen der ressourcenorientierten Stärkung der Berufswahl.

Nicht nur durch die Modifizierungen des Lehramtsstudiums, also der ersten Phase der Lehrerprofessionalisierung, kommt Dynamik in die schulische Praxis, sondern auch durch die seit einigen Jahren etablierten Programme zur Qualifizierung von Schulleiter*innen in Deutschland in der dritten Phase der Professionalisierung, die Pierre Tulowitzki, Imke Hinzen und Marvin Roller in einem bundesweiten 
Überblick dokumentieren. Gemeinsam ist den Programmen die Fokussierung von Managementfragen, Führungskompetenzen und Know-how zur Personalentwicklung. Die Qualifizierungsmaßnahmen werden jedoch in den Bundesländern mit unterschiedlicher Verbindlichkeit in die Laufbahn eingeführt. Auch hier ist eine große Dynamik zu verzeichnen, sodass etwa während der Drucklegung des Beitrags bereits Neuerungen zu verzeichnen sind, wenn - wie der Redaktion bekannt wurde nach anderen Bundesländern nun auch in Brandenburg mit der Bewerbung auf eine Schulleitungsstelle eine Qualifizierung nachgewiesen werden muss. ${ }^{1}$

Mit den sogenannten Multiplikator*innen wird im Beitrag von Bettina Fritzsche, Stephanie Schuler und Gerald Wittmann eine Gruppe von Lehrkräften in den Blick genommen, die neben ihrem Unterricht an der Schule Aufgaben der staatlichen Lehrerfortbildung übernimmt. Bislang ist wenig über das berufliche Selbstverständnis dieser Akteure bekannt; dieses Desiderat greifen die Autor*innen mit ihrer qualitativen Studie am Beispiel des Mathematikunterrichts an Grundschulen in BadenWürttemberg auf. Anhand ihrer Analysen werden Konsequenzen für die Lehrerfortbildung diskutiert.

Dass die Perspektive auf die Qualifizierung von Lehrkräften immer auch eine organisatorische Dimension hat, zeigt der Beitrag von Stephanie Niehoff, Kathrin Fussangel, Wolf-Dieter Lettau und Falk Radisch, indem sie quantitative und qualitative Befunde aus Ganztagsgrundschulen zur interprofessionellen Kooperation von Lehrkräften und pädagogischem Personal beleuchten und hierbei insbesondere die Förderangebote in den Blick nehmen. Als Herausforderung ist dies vor allem deswegen zu betrachten, da die Befunde auf eine immer noch sehr geringe Zusammenarbeit zwischen den Professionen verweisen. Eine Ausnahme stellen lediglich Fördermaßnahmen für Kinder mit sonderpädagogischem Förderbedarf dar. Hier scheint der intensive Diskurs der letzten Jahre über multiprofessionelle Zusammenarbeit im Kontext von Inklusion bereits Effekte zu zeigen. Gleichwohl müsste auch insgesamt der Ganztagsbereich von einer solchen erhöhten multiprofessionellen Kooperation profitieren.

Dass sich Kooperation hier allerdings oftmals immer noch als sperrig erweist, mag auch mit der „Eigenlogik des Nachmittags“ zusammenhängen, dessen außerunterrichtliche Angebote sich schulkulturell noch nicht immer geschmeidig in die traditionell vorherrschende Grammatik der Schule fügen. Dies zeigt sich im Abgleich der Ethnografien von Gunther Graßhoff, Christin Haude, Till-Sebastian Idel, Carolin Bebek und Anna Schütz mit den besonderen Formen des Lernens außerhalb des Unterrichts, wie sie in den Befunden der standardisierten Ganztagsschulforschung diskutiert wer-

1 Vgl. Verwaltungsvorschriften über Auswahlverfahren zur Besetzung von Leitungsfunktionen an Schulen in öffentlicher Trägerschaft im Land Brandenburg (VV-Auswahlverfahren-Leitungsfunktionen - VV-AuswahlLfkt) vom 24.02.2019. Verfügbar unter: https://bravors.bran denburg.de/verwaltungsvorschriften/vv_auswahllfkt; Zugriff am 12.03.2019. Dass dies erst mit Wirkung vom 01.03.2022 in Kraft treten wird, ist den Vorlaufmöglichkeiten geschuldet. 
den. Die ethnografische Ganztagsschulforschung differenziert damit die Perspektive auf die quantitativen Befunde, aber auch die Theoriebildung zum schulischen Ganztag.

In dem Bericht „Wiedereinstieg in den Lehrerberuf nach der Flucht mit dem Refugee Teachers Program" von Anna Aleksandra Wojciechowicz und Miriam Vock wird an der Universität Potsdam - ähnlich wie in dem Qualifizierungsprogramm „Lehrkräfte Plus“ an der Universität Bielefeld und an der Ruhr-Universität Bochum - geflüchteten Lehrkräften durch eine Nachqualifizierung die Möglichkeit eröffnet, perspektivisch in den schulisch-pädagogischen Arbeitsmarkt einzusteigen. Besondere Herausforderungen der Lehrkräftequalifizierung liegen hier oftmals sowohl in der Vermittlung der notwendigen Deutschkenntnisse als auch in den kulturell differierenden Ausbildungspraktiken und nicht zuletzt in der Berücksichtigung der individuellen Biographien, wenn zum Beispiel Gewalterfahrungen vorliegen.

Besondere biographische Lasten durch Gewalt entstehen aber auch innerhalb des deutschen Bildungssystems in zugleich ganz diffuser und doch spezifischer Weise, etwa durch sexualisierte Gewalt in schulischen Kontexten. Bernd Christmann, Daniel Schwerdt und Martin Wazlawik schärfen in ihrem Bericht die Perspektiven hierauf, indem sie Befunde der Forschung zu sexualisierter Gewalt vorstellen und auf neue Präventionskonzepte hinweisen. Jenseits von Konzepten, die auf die individuelle Lehrkraft zielen und damit auf einen spezifischen Qualifizierungsbedarf des pädagogischen Personals verweisen, werden auch organisatorisch und institutionell verankerte Präventionskonzepte diskutiert.

Die Vielfalt der Perspektiven wird konzeptionell noch einmal gesteigert, wenn wir hier abschließend gemeinsam mit Günther Wolfswinkler, Nina Bremm und Lilian Streblow nach produktiven Auswegen aus dem Paradigmenstreit suchen, der sich - teils implizit, teils explizit - im Zuge der Profilbildung an den lehrerbildenden Universitäten in den letzten Jahren gezeigt hat. Auch wenn es sich bei der in unserem Diskussionsbeitrag vorgeschlagenen Perspektive einer „multiparadigmatischen Lehrerbildung" streng genommen um eine (wissenschafts-)theoretische Unmöglichkeit handelt, da Paradigmen sich gerade dadurch auszeichnen, dass sie sich wechselseitig ausschließen und nicht kombinierbar sind, so zeigt der universitäre Alltag eben doch, dass sich die Studierenden - trotz der theoretischen Inkohärenz - in ihrem Studium unweigerlich mit unterschiedlichen Paradigmen konfrontiert sehen. Um die Studierenden hierbei nicht orientierungslos sich selbst zu überlassen, votieren wir daher für eine metaperspektivische Orientierungshilfe, die schon darin liegen könnte, dass man den Studierenden signalisiert, dass im Studium erfahrene Dissonanzen und Widersprüche nicht in individuellen Verständnisproblemen begründet liegen, sondern in der Sache einer interdisziplinären und transdisziplinären Lehrerbildung systematisch angelegt sind. 
Nach einem solchen multiperspektivischen oder auch multiparadigmatischen Durchgang durch Professionalisierungsherausforderungen in der Lehrkräftebildung dürfte zumindest eines klar sein: Es handelt sich tatsächlich um große Herausforderungen!

Wir hoffen, dass dieses Themenheft ein wenig dazu beiträgt, diese Herausforderungen zumindest besser zu erfassen, zu verstehen und sich ihnen zu stellen.

Martin Heinrich \& Isabell van Ackeren

https://doi.org/10.31244/dds.2019.02.02 